\title{
Mecanismos fisiopatológicos de la sarcopenia en la EPOC
}

\author{
WALTER SEPÚLVEDA-LOYOLA*,**, MSSC.; PAULO SERGIO** y VANESSA SUZIANE PROBST*,**, PhD.
}

\section{Pathophysiological mechanism of sarcopenia in COPD}

Sarcopenia is a disease characterized by loss of skeletal muscle, muscle strength and physical performance, being the major cause of frailty in the elderly. The sarcopenia is highly prevalent in individuals with Chronic obstructive pulmonary disease (COPD) leading to a poor prognosis and higher mortality in this population. The presence of sarcopenia in COPD is likely the result by the interaction between external and internal factors as systemic inflammation, oxidative stress and genetic polymorphisms, frequently observed in individuals with this respiratory disease. This review summarizes the current knowledge about the pathogenic mechanisms linking COPD with sarcopenia.

Key words: Sarcopenia; pulmonary disease chronic obstructive; oxidative stress; inflammation; polymorphisms genetic.

\section{Resumen}

La sarcopenia es una enfermedad caracterizada por la pérdida de masa muscular, fuerza muscular y rendimiento fisico, siendo la principal causa de fragilidad en los adultos mayores. La sarcopenia es altamente prevalente en individuos con enfermedad pulmonar obstructiva crónica (EPOC) que conduce a un mal pronóstico y una mayor mortalidad en esta población. La presencia de sarcopenia en la EPOC es probablemente el resultado de la interacción entre factores externos e internos como la inflamación sistémica, el estrés oxidativo y los polimorfismos genéticos, frecuentemente observados en individuos con esta enfermedad respiratoria. Esta revisión resume el conocimiento sobre los mecanismos patogénicos asociados con la sarcopenia en la EPOC.

Palabras clave: Sarcopenia; enfermedad pulmonar obstructiva crónica; estrés oxidativo; inflamación; polimorfismos genéticos.

* Programa de Master y Doctorado en Ciencias de la Rehabilitación, Universidad Estadual de Londrina (UEL) y Universdad Norte de Paraná (UNOPAR), Londrina, Brasil.

** Grupo de Estudio de Envejecimento (GEE), Universidad Estadual de Londrina, Brasil. 


\section{Introducción}

La enfermedad pulmonar obstructiva crónica (EPOC) es una de las principales causas de muerte en el mundo ${ }^{1,2}$, es una enfermedad prevenible y tratable, que se caracteriza por síntomas respiratorios persistentes y una limitación del flujo aéreo debido a anomalías de las vías respiratorias y/o alveolares causadas por una exposición significativa a partículas nocivas o gases ${ }^{3}$. Esta enfermedad es caracterizada por cambios extrapulmonares, como una disminución de la capacidad aeróbica, resistencia, fuerza y equilibrio, que afectan el rendimiento en las actividades de la vida diaria y la calidad de vida ${ }^{4-8}$. Además, los sujetos con EPOC tienen un nivel más bajo de actividad física, lo que puede disminuir la masa muscular y producir debilidad muscular ${ }^{9}, 10$. La debilidad muscular aumenta en presencia de enfermedades crónicas y está directamente relacionada con la sarcopenia ${ }^{11}$.

La sarcopenia ha sido definida por el grupo europeo de trabajo sobre sarcopenia en adultos mayores (EWSGOP) como un síndrome ${ }^{12}$ o una

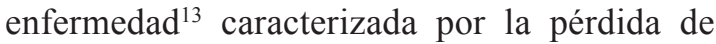
masa muscular, fuerza muscular y rendimiento físico, siendo la principal causa de fragilidad entre los ancianos ${ }^{12}$. Este síndrome tiene una prevalencia de aproximadamente $5 \%$ a $13 \%$ de todos los individuos mayores de 65 años $^{14}$. Sin embargo, en sujetos con EPOC la prevalencia de sarcopenia es aún mayor ${ }^{15,16}$.

La sarcopenia ha sido asociada a cambios metabólicos, inmovilidad, disfunción mitocondrial $^{11,17}$, estrés oxidativo ${ }^{18,19}$ e inflamación sistémica $^{11}$, que contribuyen a déficits musculares relacionados con la edad ${ }^{20}$, a través del aumento del daño celular ${ }^{18}$. En la EPOC, hay pruebas sólidas que demuestran que los cambios fisiopatológicos están relacionados con una inflamación sistémi$\mathrm{ca}^{21}$, el estrés oxidativo $(\mathrm{OS})^{22,23}$ y los polimorfismos genéticos ${ }^{24,25}$, que podrían estar asociados con la presencia acentuada de sarcopenia en esta enfermedad, ya que son los principales factores etiológicos para este síndrome ${ }^{11,18}$. Por lo tanto, esta revisión tuvo como objetivo conocer los principales mecanismos patógenos relacionados con la sarcopenia en individuos con la EPOC.

\section{Metodología}

Se realizaron búsquedas en cinco bases de datos electrónicas: PubMed, LILACS, EMBASE, Cochrane Library y SciELO desde su inicio hasta diciembre de 2018 utilizando los siguientes términos de búsqueda en inglés: "COPD", "pulmonary disease, chronic obstructive", "chronic obstructive lung disease", "COAD", "chronic obstructive airway disease" y "sarcopeni*”. En estudios que proporcionaron comparaciones entre personas con EPOC que tienen y no tienen sarcopenia, evaluamos los biomarcadores de estrés oxidativo, inflamación sistémica y polimorfismos genéticos. De los 272 registros únicos identificados a través de la búsqueda en la base de datos, 24 artículos estudiaron la sarcopenia en esta enfermedad y solo 5 de ellos determinaron biomarcadores sanguíneos en sujetos con EPOC y sarcopenia ${ }^{21,26-29}$. También fue realizada una búsqueda manual de las referencias de cada artículo incluido, para identificar otros estudios del área. Además, se utilizaron referencias complementarias de la literatura para crear una posible explicación sobre la etiología de la sarcopenia en la EPOC mediante los términos de búsqueda en inglés: "COPD", "pulmonary disease, chronic obstructive", "chronic obstructive lung disease", "COAD", "chronic obstructive airway disease", "aging" "inflammation", "oxidative stress" y "genetic polymorphisms". La información obtenida se organizó en 5 temas diferentes: 1) EPOC y sarcopenia; 2) mecanismos biológicos de la sarcopenia en la EPOC; 3 ) relación entre la inflamación y la sarcopenia; 4) relación entre el estrés oxidativo y la sarcopenia y 5) relación entre los polimorfismos genéticos y la sarcopenia.

\section{EPOC y sarcopenia}

El origen de la palabra sarcopenia deriva del griego sarx (carne) y penia (pobreza) ${ }^{30}$, por lo que al principio fue solamente relacionada a una reducción de la masa muscular. Sin embargo, hoy en día este término se ha ampliado un poco más, siendo también relacionado con la reducción de la fuerza muscular y el rendimiento físico ${ }^{13}$. Tanto ha evolucionado este concepto hoy en día, que la sarcopenia ha sido reconocida como una enfermedad musculoesquelética por la décima Revisión de la Clasificación Internacional de Enfermedades (CIE-10) en el año $2016^{31}$. Influenciado por la gran prevalencia de la sarcopenia en personas mayores de 65 años, que puede variar entre $5 \%$ y al $13 \%{ }^{14}$. También, un gran interés ha nacido por el estudio de la sarcopenia en individuos con EPOC, debido a su impacto negativo para la funcionalidad y la calidad de vida ${ }^{15,32,33}$, y su alta prevalencia que puede variar desde $15 \%$ a un $55 \%{ }^{16}$. 


\section{Mecanismos biológicos de la sarcopenia en la EPOC}

En sujetos con sarcopenia, hay un aumento en la degradación de las proteínas miofibrilares y una disminución en la síntesis de proteínas, produciendo atrofia muscular y debilidad muscu$\operatorname{lar}^{35}$. Aunque la sarcopenia es una enfermedad de los ancianos, su desarrollo puede estar asociado con otros factores, que no son exclusivamente de personas adultas mayores, como trastornos metabólicos, inflamación sistémica, estrés oxidativo, disminución de la actividad física, disfunción mitocondrial y caquexia ${ }^{18,30}$. Estos factores también están presentes en sujetos con EPOC y pueden llevar a la aparición de la sarcopenia en esta enfermedad (Figura 1) 21,22,35.

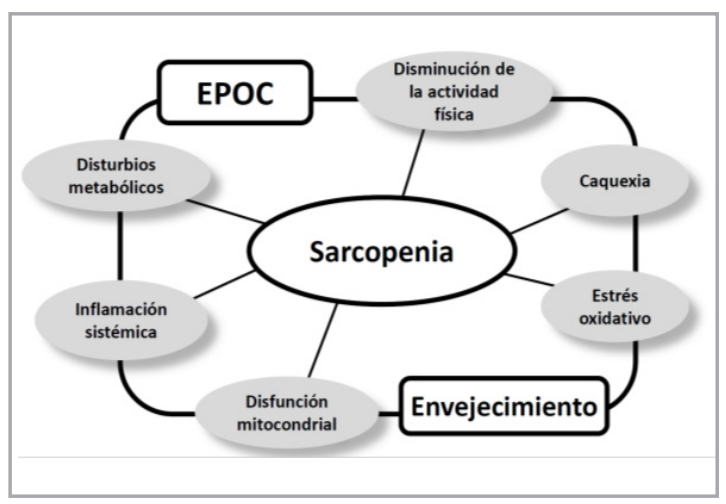

Figura 1. Características asociadas a la sarcopenia en la EPOC y el envejecimiento.
La EPOC y el envejecimiento se han relacionado con trastornos metabólicos, inflamación sistémica, disfunción mitocondrial, niveles más bajos de actividad física, caquexia y estrés oxidativo. Todos estos factores inducen la aparición de sarcopenia.

La interacción entre factores externos e internos de un individuo juegan un papel importante en el desarrollo de la sarcopenia en pacientes con EPOC. El nivel de actividad física, el tipo de dieta, el tabaquismo y el uso de corticoides producen directamente estrés oxidativo e inflamación sistémica, que combinados con polimorfismos genéticos son los responsables del origen de la sarcopenia (Figura 2) ${ }^{11,18,22,36}$. El estrés oxidativo, la inflamación crónica y la disfunción mitocondrial juegan un papel importante en la atrofia muscular, porque estos factores afectan el equilibrio entre la síntesis de proteínas y su degradación ${ }^{17,18}$. El estrés oxidativo es un desequilibrio entre las especies oxidantes y antioxidantes ${ }^{37}$. Las especies reactivas de oxígeno y nitrógeno (ROS / RNS) son segundos mensajeros para el factor de necrosis tumoral alfa (TNF- $\alpha$ ) en el músculo esquelético, activando el factor nuclear-KB (NF-kB), que induce directa e indirectamente la inflamación sistémica ${ }^{18}$. Esta inflamación sistémica induce la apoptosis celular en el músculo, que se asocia con el catabolismo muscular, disminuyendo tanto la masa muscular como la fuerza ${ }^{38}$. Esta debilidad muscular se produce a través de dos mecanismos principales: pérdida acelerada de proteínas y disfunción contráctil ${ }^{38}$.

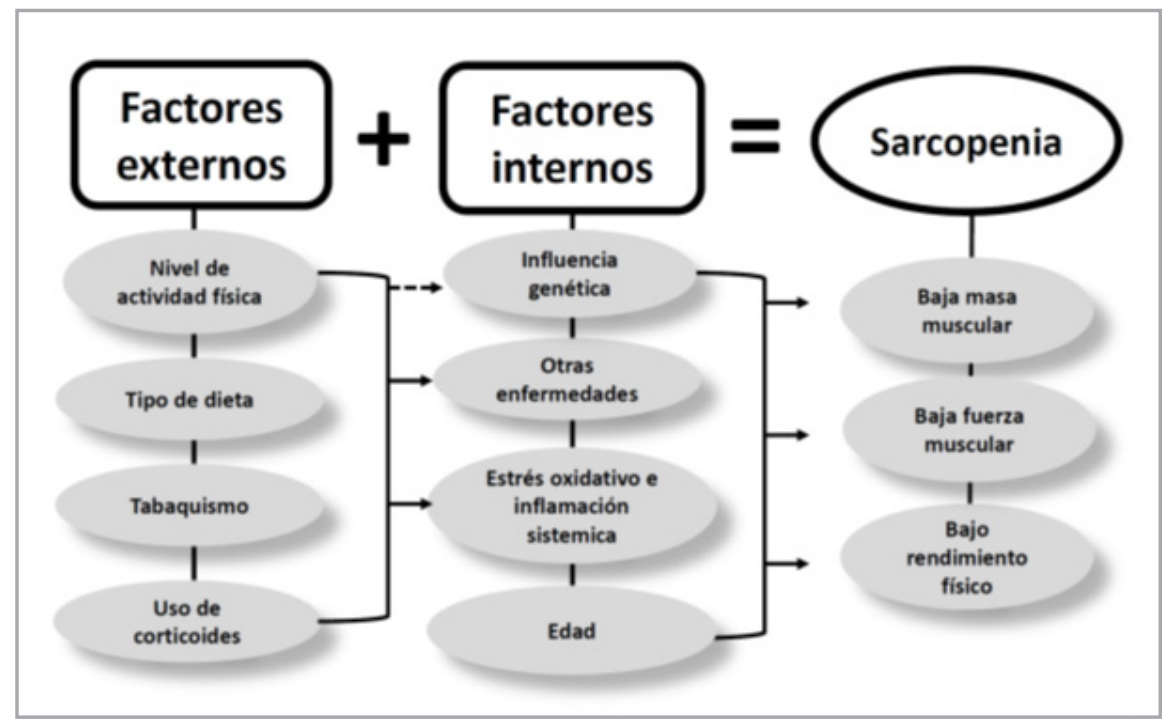

Figura 2. Factores asociados con la sarcopenia en la EPOC. La sarcopenia es inducida por factores externos e internos, que tienen asociación directa (línea continua) o asociación indirecta (línea discontinua) entre sí y con la sarcopenia. 


\section{Relación entre la inflamación y la sarcopenia}

Uno de los factores importantes asociados con la sarcopenia en las personas mayores es la inflamación sistémica ${ }^{21,39}$. La interleucina-6 (IL6), el factor de necrosis tumoral alfa y la proteína $\mathrm{C}$ reactiva (PCR) se relacionaron con una menor masa muscular, un menor rendimiento físico ${ }^{40} \mathrm{y}$ una menor fuerza muscular ${ }^{39}$ en adultos mayores. Estos biomarcadores están aumentados en individuos con EPOC comparado con aquellos sin esta enfermedad ${ }^{35,41}$. Adicionalmente, la inflamación se ha asociado con un peor estado funcional $\mathrm{y}$ de salud en individuos con EPOC ${ }^{35,41,42}$, siendo una de las posibles causas de sarcopenia en esta enfermedad ${ }^{21}$. Se han observado niveles más altos de IL- $6^{21}$, TNF- $\alpha^{21}$ y PCR ${ }^{26,29}$ en individuos con EPOC con sarcopenia en comparación con aquellos sin sarcopenia. En este contexto, la inflamación sistémica parece afectar la masa y la fuerza muscular en esta población, ya que en el estudio publicado por Byun et al. 201721, se observaron correlaciones negativas entre estas variables con los biomarcadores inflamatorios ${ }^{21}$.

Existe una fuerte relación entre la inflamación y el estrés oxidativo, por lo que estos mecanismos suelen estar vinculados ${ }^{22,43,45}$. La inflamación sistémica y el estrés oxidativo disminuyen los niveles del factor de crecimiento insulínico de tipo 1 (IGF-1) y pueden bloquear la vía de la síntesis de proteínas (Figura 3), esta reducción está asociada con sarcopenia, fragilidad y morta$\operatorname{lidad}^{25,44}$. El papel del IGF-1 es señalar la vía de proteína quinasa $\mathrm{B}$ (Akt) que induce la síntesis de proteínas mediante la estimulación de la diana de rapamicina en los mamíferos (mTOR) ${ }^{34}$. La señalización a través de IGF-1 comienza con la unión del ligando IGF-1 a su receptor, lo que induce una fosforilación del receptor y el reclutamiento del sustrato de insulina 1 (IRS1). La fosforilación de IRS1 activa la ruta del fosfoinositol-3-quinasa (PI3K), activando la Akt, la síntesis de proteínas inductora de mTOR y la hipertrofia muscular. IGF-1 es reducida durante el proceso de envejecimiento y la presencia enfermedades crónicas, disminuyendo la actividad de Akt y mTOR ${ }^{18}$.

Estas cascadas señalizadas por biomarcadores inflamatorios y estrés oxidativo son activadas por el proceso de envejecimiento y son estimuladas en presencia de enfermedades crónicas, como la EPOC, lo cual está asociado con sarcopenia (Figura 3) ${ }^{22,35}$.

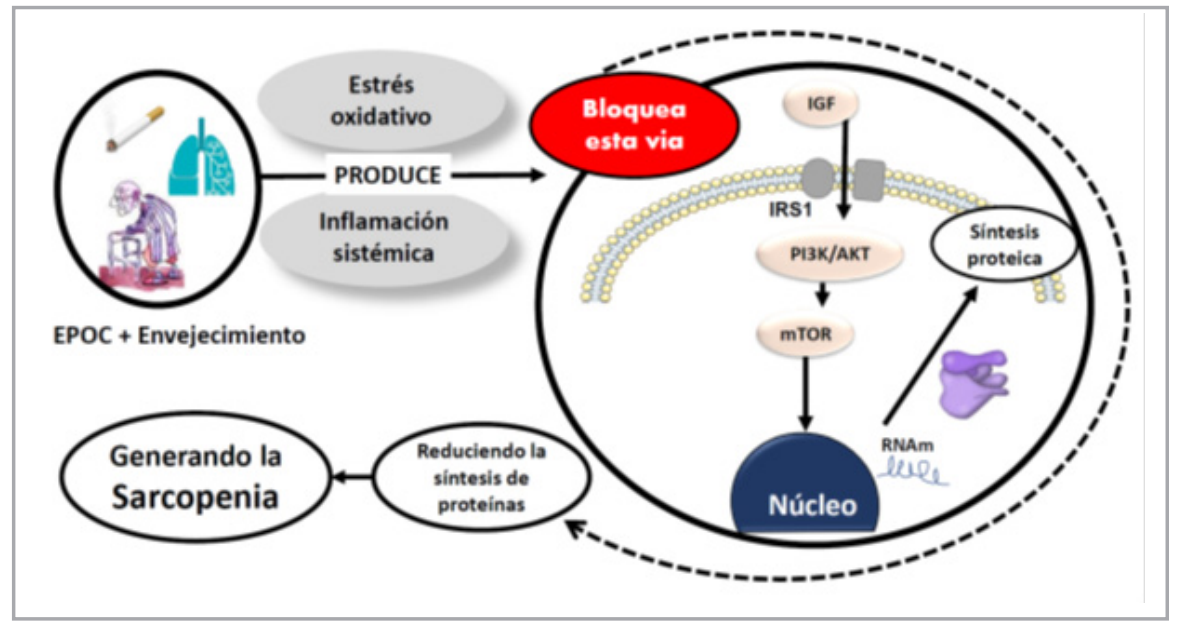

Figura 3. La EPOC combinada con el envejecimiento generan la sarcopenia. La EPOC y el envejecimiento están asociados con la inflamación sistémica y el estrés oxidativo, los cuales bloquean la vía IGF-1 / PI3K / AKT / mTOR, lo que reduce la síntesis de proteínas musculares, por lo tanto, induce la sarcopenia.

\section{Relación entre el estrés oxidativo y la sarcopenia}

Cesari et al. ${ }^{46}$, demostraron una correlación positiva entre la capacidad antioxidante con el rendimiento físico y la fuerza muscular en ancianos. Estos autores mostraron que los sujetos con mayor ingesta de antioxidantes en la dieta, como la vitamina $\mathrm{C}$, son los que tienen mayor fuerza muscular. Además, Agler et al. ${ }^{47}$, en un estudio de seguimiento de 10 años con 38.597 mujeres ( $\geq 45$ años) encontraron que la suplementación con antioxidantes redujo el riesgo de desarrollar enfermedad pulmonar crónica en un 10\% (HR 0,90 ; IC del 95\%: 0,81 a 0,99; $p=0,029$ ), adicionalmente se asoció con una disminución de los niveles de estrés carbonilo en el pulmón. Los estudios en individuos con EPOC demostraron una reducción en la actividad antioxidante de la 
superóxido dismutasa (SOD), catalasa (CAT), proteínas sulfhidrilas totales $(\mathrm{SH})$, paraoxonasa 1 (PON1), oxidasa y glutatión-Stransferasa (GST) ${ }^{48-51}$. Si embargo, hay resultados contradictorios con algunos biomarcadores antioxidantes, como $\mathrm{SH}$, superóxido dismutasa y catalasa, que han demostrado un aumento o no diferencias, en comparación con los individuos sin la EPOC $^{52,53}$.

Los sujetos con EPOC tienen un desequilibrio entre los niveles de oxidantes y antioxidantes ${ }^{48-50}$. Los niveles de biomarcadores oxidantes están aumentados en individuos con $\mathrm{EPOC}^{48-56}$. Ia peroxidación lipídica y la oxidación de proteínas son consecuencias del estrés oxidativo y causan daño oxidativo ${ }^{50,52,57,58}$. Se ha demostrado, que este daño oxidativo estaría relacionado con un deterioro en la función pulmonar, gravedad de la enfermedad y la presencia de síndrome metabólico en individuos con $\mathrm{EPOC}^{23}$.

Las sustancias reactivas al ácido tiobarbitúrico (TBARS) y el malondialdehído (MDA) son los biomarcadores más comunes de la peroxidación de lípidos estudiados en sujetos con EPOC $^{50}$. Niraj Dhakal et al. ${ }^{50}$, Raut et al. ${ }^{55}$ y Wozniak et al. ${ }^{53}$, informaron niveles más altos de MDA y TARBS en sujetos con EPOC en comparación con el grupo control. Por otro lado, Syrine et al. ${ }^{56}$ y Jammes et al..$^{59}$, no observaron diferencias en los niveles de MDA y TBARS, respectivamente, entre los individuos con EPOC y aquellos sin esta enfermedad. Además, el aldehído 4-hidroxi2-nonenal (4-HNE), el principal compuesto de la peroxidación lipídica de la membrana, está elevado en individuos con EPOC, que es un mediador clave en diferentes mecanismos relacionados con el estrés oxidativo, la inflamación y la apoptosis celular ${ }^{57,58}$.

Con respecto a la oxidación de proteínas, en individuos con EPOC, el grado del daño oxidativo en las proteínas se ha investigado a través de la presencia de productos de oxidación avanzada de proteínas (AOPP) ${ }^{17}$. Stanojkovic et al..$^{60}$, encontraron niveles más altos de AOPP y MDA en sujetos con EPOC en comparación con individuos aparentemente sanos, además, este estudio mostró que la inflamación sistémica elevada está correlacionada negativamente con la capacidad antioxidante. Por lo tanto, estos resultados enfatizan la relación entre el estrés oxidativo y la inflamación.

Existe escasa evidencia sobre los marcadores de estrés oxidativo y la sarcopenia en individuos con $\mathrm{EPOC}^{56}$. La mayoría de los estudios correlacionaron los biomarcadores del estrés oxidativo con el índice de masa corporal, la función pul- monar, el estado nutricional, las exacerbaciones, estadios de GOLD y la inflamación sistémi$\mathrm{ca}^{23,50,56,60-62}$ La investigación de Byun et $\mathrm{al}^{21}$ es la unica evidencia que ha informado una asociación entre algunos biomarcadores inflamatorios con sarcopenia en sujetos con EPOC. Aunque no hay evidencia de asociación entre la sarcopenia y el estrés oxidativo en esta población, podemos suponer que el estrés oxidativo desempeña un papel importante en el origen de la sarcopenia, ya que el estrés oxidativo está vinculado a diferentes mecanismos biológicos relacionados con la inflamación y la atrofia muscular ${ }^{50,52,57,58,60}$.

\section{Relación entre los polimorfismos genéticos y la sarcopenia}

La influencia de la genética en la sarcopenia, ha sido estudiada recientemente ${ }^{44}$. Los polimorfismos de un solo nucleótido (SNP) están relacionados con la inflamación sistémica y la disminución de la síntesis de proteínas, por lo que se han asociado con la sarcopenia y la fragilidad en los adultos mayores ${ }^{63}$. Las mutaciones en los genes IGF-I e IGF-II en seres humanos reducen la síntesis de IGF, esto se ha relacionado a fenotipos caracterizados por menor fuerza muscular, menor masa muscular y una menor respuesta al entrenamiento con ejercicios ${ }^{64,65}$. El C-1245T SNP (rs35767) es una variación genética en la región promotora del IGF-I, que se ha estudiado en ancianos, asociada con disminuciones de la masa muscular y la fuerza muscular, ya que el gen IGFI regula la síntesis de proteínas del músculo y la hipertrofia ${ }^{65,66}$. Además, las mutaciones genéticas pueden interferir en la respuesta del ejercicio.

Devaney et $\mathrm{al}^{64}$, encontraron que el polimorfismo de IGF-II (rs3213221) produce una mayor pérdida de fuerza muscular, mayor dolor y aumento en los niveles de la creatina quinasa sérica posterior al ejercicio, por lo que este gen se ha relacionado con mayor riesgo de daño muscular ${ }^{66}$. Otro polimorfismo asociado con sarcopenia y fragilidad es la mutación en el gene ACTN-3 en humanos (rs1815739), lo que puede disminuir el nivel de $\alpha$-Actinina-3, alterando la función contráctil del músculo, debido a que es una proteína fundamental en la contracción muscular ${ }^{66}$.

Como fue explicando anteriormente, los biomarcadores proinflamatorios desempeñan un papel en la debilidad muscular ${ }^{18}$. La producción de citoquinas inflamatorias puede estar influenciada por polimorfismos genéticos, generalmente en la región promotora de los genes ${ }^{67}$. De este modo, tanto la sarcopenia como la inflamación sistémica pueden verse influidas por polimorfismos genéticos. En este sentido, Broekhuizen et al. ${ }^{68}$, 
encontraron una mayor prevalencia del polimorfismo IL-1 $\beta-511$ en individuos con EPOC con menor índice de masa magra en comparación con sujetos aparentemente sanos. Estos hallazgos sugieren una predisposición genética en el proceso de caquexia en sujetos con $\mathrm{EPOC}^{68}$.

Finalmente, la contribución genética al desarrollo de la EPOC ha sido bien establecida en una revisión sistemática desarrollada por Bossé et $\mathrm{al}^{71}$. Sin embargo, hay pocos estudios que muestren correlación entre la presencia de sarcopenia en la EPOC con factores genéticos. Además, la mutación de los genes ILG-II e IGF-II ha sido poco estudiada en individuos con EPOC, ya que la mayoría de los polimorfismos de un solo nucleótido (SNP) asociados con esta enfermedad se han relacionado con la inflamación sistémica y la función pulmonar ${ }^{69}$.

\section{Conclusiones}

La interacción entre factores externos e internos como la inflamación sistémica, el estrés oxidativo y los polimorfismos genéticos están relacionados con la prevalencia de la sarcopenia en individuos con la EPOC. Sin embargo, son necesarios nuevos estudios para identificar algunos biomarcadores sanguíneos y genéticos específicos relacionados con la fuerza muscular, la masa muscular y el rendimiento físico en esta enfermedad.

\section{Bibliografía}

1.- HALBERT RJ, NATOLI JL, GANO A, BADAMGARAV E, BUIST AS, MANNINO DM. Global burden of COPD: systematic review and meta-analysis. Eur Respir J. 2006; 28: 523-532. doi:10.1183/09031936.06.001246 05 .

2.- QUADERI SA, HURST JR. The unmet global burden of COPD. 2018: 82-4. doi:10.1017/gheg. 2018.1

3.- Global Strategy for the Diagnosis, Management and Prevention of COPD, Global Initiative for Chronic Obstructive Lung Disease (GOLD) 2017; doi:10.1097/00008483-200207000-00004

4.- ROIG M, ENG J, MACINTYRE D, ROAD J, REID W. Falls in patients with chronic obstructive pulmonary disease: a call for further research. Respir Med. 2009; 103(9): 1257-69. doi: 10.1016/j.rmed. 2009.03.022.

5.- ANNEGARN J, MEIJER K, PASSOS VL, STUTE K, WIECHERT J, SAVELBERG MH, et al. Problematic activities of daily life are weakly associated with clinical characteristics in COPD. J Am Med Dir Assoc. 2012; 13: -90. doi: 10.1016/j.jamda.2011.01.002.

6.- WELDAM SWM, SCHUURMANS MJ, LIU R, LAM-
MERS J-WJ. Evaluation of Quality of Life instruments for use in COPD care and research: A systematic review. Int J Nurs Stud. 2013; 50: 688-707. doi:10.1016/j.ijnurstu.2012.07.017.

7.- TAKEDA K, KAWASAKI Y, YOSHIDA K, NISHIDA P, MARADA T, YAMAGUCHI K, et al. The 6-minute pegboard and ring test is correlated with upper extremity activity of daily living in chronic obstructive pulmonary disease. Int J Chron Obstruct Pulmon Dis. 2013; 8: 347-51. doi: 10.2147/COPD.S45081.

8.- JANSON C, MARKS G, BUIST S, GNAHUC L, GILASON T, MC BURNIC M, al. The impact of COPD on health status: Findings from the BOLD study. Eur Respir J. 2013; 42: 1472-83. doi: 10.1183/09031936.00153712.

9.- HILL K, GOLDSTEIN RS. Limited functional performance in chronic obstructive pulmonary disease: nature, causes and measurement. COPD. 2007; 4: 257-61. doi:10.1080/15412550701480224.

10- BURTIN C, TER RIET G, PUHAN MA, WASCHKI B, GARCIA-AYMERICH J, PINTO-PLATA V, et al. Handgrip weakness and mortality risk in COPD: a multicentre analysis. Thorax. 2016; 71: 86-7. doi: 10.1136/ thoraxjnl-2015-207451.

11.- ROLLAND Y, CZERWINSKI S, ABELLAN VAN KAN G, MORLEZ SE, CESARI M, ONDER G, et al. Sarcopenia: its assessment, etiology, pathogenesis, consequences and future perspectives. J Nutr Health Aging. 2011; 12: 433-50. doi: 10.1126/scisignal.2001449. Engineering.

12.- CRUZ-JENTOFT AJ, BAEYENS JP, BAUER JM, BOIRIE Y, CEDERHOLM T, LANDI F, et al. Sarcopenia: European consensus on definition and diagnosis. Age Ageing. 2010; 39: 412-23. doi:10.1093/ageing/ afq034.

13.- CRUZ-JENTOFT AJ, BAHAT G, BAUER J, BOIRIE Y, BRUYĖRE O, CEDERHOLM T, et al. Sarcopenia: revised European consensus on definition and diagnosis. Age Ageing. 2018; 1: 1-16. doi:10.1093/ageing/afy169.

14.- SANTILLI V, BERNETTI A, MANGONE M, PAOLONI M. Clinical definition of sarcopenia 2013.pdf. Clin Cases Miner Bone Metab. 2014; 11: 177-80. doi: 1138/ $\mathrm{ccmbm} / 2014.11 .3 .177$.

15.- JONES SE, MADDOCKS M, KON SSC, CANAYAN JL, NOLAN CM, CLARK AL, et al. Sarcopenia in COPD: prevalence, clinical correlates and response to pulmonary rehabilitation. Thorax. 2015; 70 213-18. doi:10.1136/thoraxjnl-2014-206440

16.- COSTA TM DA RL, COSTA FM, MOREIRA CA, RABELO LM, BOGUSWESKI, BORBA VZ, et al. Sarcopenia in COPD: relationship with COPD severity and prognosis. J Bras Pneumol. 2015; 41: 415-21. doi:10.1590/S1806-37132015000000040.

17.- MARZETTI E, CALVANI R, CESARI M, BUFORD TW, LORENZI M, BEHNKE BJ, et al. Mitochondrial dysfunction and sarcopenia of aging: From signaling 
pathways to clinical trials. Int J Biochem Cell Biol. 2013; 45: 2288-301. doi:10.1016/j.biocel.2013.06.024.

18.- MENG SJ, YU LJ. Oxidative stress, molecular inflammation and sarcopenia. Int J Mol Sci. 2010; 11: 150926. doi:10.3390/ijms11041509.

19.- JACKSON MJ. Reactive oxygen species in sarcopenia: Should we focus on excess oxidative damage or defective redox signalling? Mol Aspects Med. 2016; 50: 33-40. doi:10.1016/j.mam.2016.05.002.

20.- TOBERGTE DR, CURTIS S. Age-induced oxidative stress: how does it influence skeletal muscle quantity and quality? J Chem Inf Model. 2013; 53 :1689-99. doi:10.1017/CBO9781107415324.004.

21.- BYUN, MIN KWANG, EUN NA CHO, JOON CHANG, CHUL MIN AHN HJK. Sarcopenia correlates with systemic inflammation in COPD. Int J Chron Obs Pulmon Dis. 2017: 669-75.

22.- KIRKHAM PA, BARNES PJ. Oxidative stress in COPD. Chest. 2013; 144: 266-73. doi:10.1378/ chest.12-2664.

23.- SEPÚLVEDA LOYOLA WA, VILAÇA CAVALLARI MACHADO F, ARAÚJO DE CASTRO L, HIBBNAVER LEAL BALTHUS T, RAMPAZZO MORELLI $\mathrm{L}$, LAMDUCCI BONIFACIO $\mathrm{K}$, et al. Is oxidative stress associated with disease severity, pulmonary function and metabolic syndrome in chronic obstructive pulmonary disease? Rev Clin Esp. 2019; Jun 29; doi: 10.1016/j.rce.2019.04.007.

24.- BEN-ZAKEN S, MECKEL Y, NEMET D, ELIAKIM A. Can IGF-I polymorphism affect power and endurance athletic performance? Growth Horm IGF Res. 2013; 23: 175-8. doi:10.1016/j.ghir.2013.06.005.

25.- BARBIERI M, FERRUCCI L, RAGNO E, et al. Chronic inflammation and the effect of IGF-I on muscle strength and power in older persons. Am J Physiol Endocrinol Metab. 2003; 284: E481-E487. doi:10.1152/ ajpendo.00319.2002.

26.- VAN DE BOOL C, RUTTEN EPA, FRANSSEN FME, WOUTERS EFM, SCHOLS AMWJ. Antagonistic implications of sarcopenia and abdominal obesity on physical performance in COPD. Eur Respir J. 2015; 46: 336-45. doi: 10.1183/09031936.00197314.

27.- JOPPA P, TKACOVA R, FRANSSEN FME, HANSON C, RENNARD SI, SILVERMAN EK, et al. Sarcopenic Obesity, Functional Outcomes, and Systemic In fl ammation in Patients With Chronic Obstructive Pulmonary Disease. J Am Med Dir Assoc. 2016; 17: 712-18. doi:10.1016/j.jamda.2016.03.020.

28. CEBRON LIPOVEC N, SCHOLS AMWJ, VAN DEN BORST B, BEIJERS RJ, KORSTEN T, OMERSAD, et al. Sarcopenia in Advanced COPD Affects Cardiometabolic Risk Reduction by Short-Term High-intensity Pulmonary Rehabilitation. J Am Med Dir Assoc. 2016; 17: 814-20. doi: 10.1016/j.jamda.2016.05.002.

29.- DE BLASIO F, DI GREGORIO A, DE BLASIO F, BRANCO BELLOFIORE B, SCALFI L, et al. Mal- nutrition and sarcopenia assessment in patients with chronic obstructive pulmonary disease according to international diagnostic criteria, and evaluation of raw BIA variables. Respir Med. 2018; 134(July 2017): 1-5. doi:10.1016/j.rmed.2017.11.006.

30.- MUSCARITOLI M, ANKER S.D., ARGILES J, AVERSA Z, BAUER JM, BIOLOG, et al. Consensus definition of sarcopenia, cachexia and pre-cachexia : Joint document elaborated by Special Interest Groups ( SIG ) "“ cachexia-anorexia in chronic wasting diseases "” and "“ nutrition in geriatrics ." Clin Nutr. 2010; 29: 154-9. doi:10.1016/j.clnu.2009.12.004.

31.- ANKER SD, MORLEY JE, VON HAEHLING S. Welcome to the ICD-10 code for sarcopenia. J Cachexia Sarcopenia Muscle. 2016; 7: 512-4. doi:10.1002/ jcsm.12147.

32.- LIMPAWATTANA P, INTHASUWAN P, PUTRAVEEPHONG S, BOONSAWAT W, THEERAKULPISUT D, SAWANYAWISUTH K. Sarcopenia in chronic obstructive pulmonary disease: A study of prevalence and associated factors in the Southeast Asian population. Chron Respir Dis. 2017; 147997231774375. doi:10.1177/1479972317743759.

33.- COSTA TM DA RL, COSTA FM, MOREIRA CA, RABELO LM, BOGUSZEWSKI CL, BORBA VZC. Sarcopenia in COPD: relationship with COPD severity and prognosis TT - Sarcopenia na DPOC: relação com a gravidade e o prognóstico da DPOC. J Bras Pneumol. 2015; 41: 415-21. Disponible en: http:// www.scielo.br/scielo.php?script=sci_arttext\&pid $=$ S1806-37132015000500415.

34.- YOSHIDA T, DELAFONTAINE P. Mechanisms of Cachexia in Chronic Disease States. Am J Med Sci. 2015; 350: 250-256. doi:10.1097/MAJ.0000000000000511.

35.- EAGAN TML, AUKRUST P, UELAND T, HARDIE JA, JOHANNESSEN A. Body composition and plasma levels of inflammatory biomarkers in COPD. Eur Respir J. 2010; 36: 1027-33. doi:10.1183/09031936.00194209.

36.- ROTH SM. Genetic aspects of skeletal muscle strength and mass with relevance to sarcopenia. Bonekey Rep. 2012; 1: 1-7. doi:10.1038/bonekey.2012.58.

37.- KREGEL KC, ZHANG HJ. An integrated view of oxidative stress in aging: basic mechanisms, functional effects, and pathological considerations. Am J Physiol Regul Integr Comp Physiol 292. 2007; 292: 18-36. doi:10.1152/ajpregu.00327.2006.

38.- CARTER CS, HOFER T, SEO AY, LEEUWENBURGH C, KIKNOW DTBL, VON ZEWICKI T. Molecular mechanisms of life- and health-span extension: role of calorie restriction and exercise intervention. Appl Physiol Nutr Metab. 2007; 966: 954-66. doi:10.1139/ H07-085.

39.- GRANIC A, DAVIES K, MARTIN-RUIZ C, JAGGER $\mathrm{C}$, et al. Grip strength and inflammatory biomarker profiles in very old adults. Age Ageing. 2017; 46: 976-82. doi:10.1093/ageing/afx088. 
40.- KIM D-Y, KIM C-O, LIM H. Quality of diet and level of physical performance related to inflammatory markers in community-dwelling frail, elderly people. Nutrition. 2017; 38: 48-53. doi:10.1016/j.nut.2016.12.023.

41.- GERRITSEN WBM, ASIN J, ZANEN P, VANDEN BOCH JM, HAAS FJ, Markers of inflammation and oxidative stress in exacerbated chronic obstructive pulmonary disease patients. Respir Med. 2005;99:84-90. doi:10.1016/j.rmed.2004.04.017

42.- HALLIN R, JANSON C, ARNARDOTTIR RH, et al. Relation between physical capacity, nutritional status and systemic inflammation in COPD. Clin Respir J. 2011; 5: 136-42. doi:10.1111/j.1752699X.2010.00208.x.

43.- LIGUORI I, RUSSO G, CURCIO F, BULLI G, ARAN L, DELLA-MORTE D, et al. Oxidative stress, aging, and diseases. Clin Interv Aging. 2018;13:757-72. doi:10.2147/CIA.S158513

44.- MCKEE A, MORLEY JE, MATSUMOTO AM, VINIK A. Sarcopenia an endocrine Disorder? Endocr Pract. 2017; 23: 1140-9. doi:10.4158/EP171795.RA.

45.- BARNES PJ. Inflammatory mechanisms in patients with chronic obstructive pulmonary disease. J Allergy Clin Immunol. 2016; 138: 16-27. doi:10.1016/j. jaci.2016.05.011.

46.- CESARI M, PAHOR M, BARTALI B, CHERUBINI A, PENNINX PW, WILLIAMS GR, et al. Antioxidants and physical performance in elderly persons : the Invecchiare in Chianti ( InCHIANTI ) study 1 - 3. Am J Clin Nutr. 2004; 79: 289-94.

47.- AGLER AH, KURTH T, GAZIANO JM, BURING JE, CASSANO A. Randomised Vitamin E Supplementation and Risk of Chronic Lung Disease in the Women's Health Study. Thorax. 2011; 66: 320-5. doi:10.1136/ thx.2010.155028.Randomised.

48.- NADEEM A, RAJ HG, CHHABRA SK. Increased Oxidative Stress and Altered Levels of Antioxidants in Chronic Obstructive Pulmonary Disease. Inflammation. 2005; 29: 23-4. doi:10.1007/s10753-006-8965-3.

49.- AHMAD A, SHAMEEM M, HUSAIN Q. Altered oxidant-antioxidant levels in the disease prognosis of chronic obstructive pulmonary disease. Int $\mathrm{J}$ Tuberc Lung Dis. 2013; 17: 1104-9.

50.- DHAKAL N, LAMSAL M, BARAL N, SHRESTHA S. Oxidative Stress and Nutritional Status in Chronic Obstructive Pulmonary Disease. J Clin Diagn Res. 2015; 2: BC01-4. doi:10.7860/JCDR/2015/9426.5511.

51.- RUMORA L, RAJKOVIĆ MG, KOPČINOVIĆ LM, PANCIROV D, ČEPELAK I GT. Paraoxonase 1 Activity in Patients with Chronic Obstructive. COPD J Chronic Obstr Pulm Dis. 2014; 539-45. doi:10.3109/15 412555.2014.898028.

52.- ZINELLU E, ZINELLU A, FOIS AG, CARRU C, PIRINA P. Circulating biomarkers of oxidative stress in chronic obstructive pulmonary disease: a systematic review. Respir Res. 2016; 17: 150. doi:10.1186/s12931- 016-0471-z

53.- WOZNIAK A, GÓRECKI D, SZPINDA MB, MILAKIERZENKOWSKA C, WO B. Oxidant-Antioxidant Balance in the Blood of Patients with Chronic Obstructive Pulmonary Disease After Smoking Cessation. Oxid Med Cell Longev. 2013; 2013: 89707.

54.- PINHO RA, CHIESA D, MEZZOMO KM, ANDRADES ME, BONATTO F, GELAIN D, et al. Oxidative stress in chronic obstructive pulmonary disease patients submitted to a rehabilitation program. Respir Med. 2007; 101: 1830-5. doi:10.1016/j.rmed.2007.02.004.

55.- RAUT AM, SURYAKAR AN, MHAISEKAR D. A study of oxidative stress, thiol proteins and role of vitamin E supplementation in chronic obstructive pulmonary disease ( COPD ). Al Am een J Med Sci. 2013; 6: 134-7.

56.- MOUSSA S BEN, SFAXI I, TABKA Z, SAAD H BEN, ROUATBI S. Oxidative stress and lung function profiles of male smokers free from COPD compared to those with COPD: a case-control study. Libyan J Med. 2014; 1: 1-13.

57.- RAHMAN I, VAN SCHADEWIJK AAM, CROWTHER AJL, et al. 4-Hydroxy-2-nonenal, a specific lipid peroxidation product, is elevated in lungs of patients with chronic obstructive pulmonary disease. Am J Respir Crit Care Med. 2002; 166: 490-5. doi:10.1164/ rccm.2110101

58.- TAKIMOTO T, YOSHIDA M, HIRATA H, KASHIWA Y, TAKEDA Y, GOYA S, et al. 4-Hydroxy-2-nonenal induces chronic obstructive pulmonary disease-like histopathologic changes in mice. Biochem Biophys Res Commun. 2012; 420: 84-90. doi:10.1016/j. bbrc.2012.02.119.

59.- JAMMES Y, STEINBERG JG, BA A, DELLIAUX S, BREGEON F. Enhanced exercise-induced plasma cytokine response and oxidative stress in COPD patients depend on blood oxygenation. Clin Physiol Funct Imaging. 2008; 3: 182-8. doi:10.1111/j.1475097X.2008.00795.x.

60.- STANOJKOVIC I, KOTUR-STEVULJEVIC J, MILENKOVIC B. Pulmonary function, oxidative stress and inflammatory markers in severe COPD exacerbation. Respir Med. 2011: 105 Suppl: 31-37. doi:10.1016/ S0954-6111(11)70008-7.

61.- VIBHUTI A, ARIF E, DEEPAK D, SINGH B, PASHA MAQ. Correlation of oxidative status with BMI and lung function in COPD. Clin Biochem. 2007; 40: 95863. doi:10.1016/j.clinbiochem.2007.04.020.

62.- ANES A, FETOUI H, BCHIR S, NASR H, CHAHDOURA H. Increased Oxidative Stress and Altered Levels of Nitric Oxide and Peroxynitrite in Tunisian Patients with Chronic Obstructive Pulmonary Disease : Correlation with Disease Severity and Airflow Obstruction. Biol Trace Elem Res. 2014; 161: 20-31. doi:10.1007/s12011-014-0087-4.

63.- VIÑA J, TARAZONA-SANTABALBINA FJ, PÉREZROS P, et al. Biology of frailty: Modulation of ageing 
genes and its importance to prevent age-associated loss of function. Mol Aspects Med. 2016; 50: 88-108. doi:10.1016/j.mam.2016.04.005.

64.- DEVANEY JM, HOFFMAN EP, GORDISHDRESSMAN H, KEARNS A, ZAMBRASKI E, CLARKSON PM. IGF-II gene region polymorphisms related to exertional muscle damage. J Appl Physiol. 2007; 102: 1815-23. doi:10.1152/japplphysiol.01165.2006.

65.- KIKUCHI N, YOSHIDA S, MIN S, LEE K, SKAMAKI -SUMAGA M, OKAMOTO T, et al. The ACTN3 $\mathrm{R} 577 \mathrm{X}$ genotype is associated with muscle function in a Japanese population. Appl Physiol Nutr Metab. 2015; 40: 316-22. doi:10.1139/apnm-2014-0346.

66.- BAUMERT P, LAKE MJ, STEWART CE, DRUST B, ERSKINE RM. Genetic variation and exercise-induced muscle damage: implications for athletic performance, injury and ageing. Eur J Appl Physiol. 2016; 116: 1595 1625. doi:10.1007/s00421-016-3411-1-

67.- PAOLONI-GIACOBINO A, GRIMBLE R, PICHARD C. Genomic interactions with disease and nutrition. Clin Nutr. 2003; 22: 507-14. doi:10.1016/S02615614(03)00091-8.

68.- BROEKHUIZEN R, GRIMBLE RF, HOWELL WM, SHALE DJ, CREUTZBERG EC, WOUTERS EF, et al. Pulmonary cachexia, systemic inflammatory profile, and the interleukin 1 beta-511 single nucleotide polymorphism. Am J Clin Nutr. 2005; 82: 1059-64. doi:82/5/1059 [pii].

69.- BOSSÉ Y. Updates on the COPD gene list. Int J COPD. 2012; 7: 607-31. doi:10.2147/COPD.S35294.

Correspondencia a:

Walter Aquiles Sepúlveda Loyola

Av. Robert Koch. 60- Vila Operária

CEP: 86038-350

Londrina-Paraná-Brasil.

Email: walterkine2014@gmail.com 